\title{
Some remarks on the density of regular mappings in Sobolev classes of $S^{M}$-valued functions
}

\author{
MigúEl ESCOBEDO
}

ABSTRACT. Some results are given about the density of continuous maps from $\Omega$, a bounded regular domain of $\mathbb{R}^{N}$ to $S^{M}$ in the Sobolev classes $W^{s, P}\left(\Omega, S^{M}\right)$.

\section{INTRODUCTION}

We are interested in the following question: suppose that $\Omega$ is a regular domain of $\mathbb{R}^{N}$ and $\mathfrak{N}$ is a submanifold of $\mathbb{R}^{M+1}$. Consider, for two real numbers $p \geqslant 1$, and $s \geqslant 0$, any vector valued function $u$ of the Sobolev space $W^{s, p}\left(\Omega, \mathbb{R}^{M+1}\right)$ taking its values in $\mathfrak{g}$. Is it possible to approximate $\mathrm{u}$ in the space $W^{s, p}\left(\Omega, \mathbb{R}^{M+1}\right)$ by regular functions taking also their values in $\mathfrak{n}$ ? Or equivalently is the following subset of $C^{\infty}\left(\Omega, R^{M+1}\right)$ :

$$
C^{\infty}(\Omega, \mathfrak{N})=\left\{u \in C^{\infty}\left(\Omega, \mathbb{R}^{M+1}\right) ; \forall x \in \Omega, u(x) \in \mathfrak{R}\right\}
$$

dense in the subset of $W^{s, p}\left(\Omega, \mathbb{R}^{M+1}\right)$ :

$$
W^{s, p}(\Omega, \mathfrak{N})=\left\{u \in W^{s, p}\left(\Omega, \mathbb{R}^{M+1}\right) ; \forall x \in \Omega, u(x) \in \mathfrak{R}\right\} ?
$$

First of all observe that none of these sets are vector spaces. In particular smoothing functions of $W^{s, p}(\Omega, \mathfrak{N})$ by taking mean values on balls or by convolution produces functions whose values do not lie in $\mathfrak{\Re}$.

It is known that when $p \geqslant N, C\left(\Omega, S^{M}\right) \cap W^{1, p}\left(\Omega, S^{M}\right)$ (where $S^{M}$ is the unit sphere of $\left.\mathbb{R}^{M+1}\right)$ is dense in $W^{1, p}\left(\Omega, S^{M}\right)$ and this for all values of the integer $M$. (See [2]. [10]. [12].) If $p<N$ this result is no longer true and in fact the relation between $p$ and $M$ turns out to be determinant. For example, if $M$ is less than $p$ it may be that there is no density as it is shown in [4], [10] and [12]. On the other hand, H. Brezis knew how to prove density result for the

1980 Mathematics Subject Classification (1985 revision): 46E35.

Editorial de la Universidad Complutense. Madrid, 1988. 
space $L^{P}\left(\Omega, S^{\prime \prime}\right)$ for every $p \geqslant 1$ using stereographic projection. Using similar ideas, F. Bethuel and D. Zheng in [5] have proved the density in the space $W^{1, p}\left(\Omega, S^{n}\right)$ when $p<M$. The way to do this is to approximate functions of $W^{1, p}\left(\Omega, S^{M}\right)$ in two steps. First by functions of $W^{1, p}\left(\Omega, S^{M}\right)$ not necessarily continuous but taking their values on a segment of the sphere $S^{M}$. Secondly, since any segment of a sphere $S^{M}$ is diffeomorphic to $\mathrm{R}^{M}$ it is rather simple to approximate any function of $W^{1, p}\left(\Omega, S^{M}\right)$, taking values on a segment of sphere, by functions of $C^{\infty}\left(\Omega, S^{w}\right)$.

This is also what we do in the more general case of the sets $W^{m, p}\left(\Omega, S^{M}\right)$, $m$ integer and $\Lambda_{p q}^{s}\left(\Omega, S^{M}\right), s$ non negative real. Our main results are:

Theorem 1. Given two integers $M, N$ and three reals $s>0, \infty>p \geqslant 1$ and $\infty>q \geqslant 1$ such that $s p \geqslant N$ the set $C\left(\Omega, S^{M}\right) \cap \Lambda_{p q}^{s}\left(\Omega, S^{M}\right)$ is dense in $\Lambda_{p q}^{s}\left(\Omega, S^{M}\right)$.

Theorem 2. Given three reals $\infty>p \geqslant 1, \infty \geqslant q \geqslant 1, s \geqslant 0$ and two integers $N$, $M$ such that $s p<N$, if: $\max (1, s) \cdot \max (p, q)<M$, or $0<s<1 / p$ and $1+N / p>s+N / q$, then the set $C^{x}\left(\Omega, S^{M}\right)$ is dense in $\Lambda_{p q}^{s}\left(\Omega, S^{M}\right)$.

Theorem 3. If 9 is a compact Riemann manifold of dimension $M, B^{N}(0,1)$ is the unit ball of $\mathbb{R}^{N} s>0$ and $p \geqslant 1$ are two reals such that $s p<N$ and the [sp]-th homotopy group of $\mathfrak{\Re ,} \Pi_{|s p|}(\mathfrak{P})$, is not the trivial one then $C\left(B^{N}(0,1), \mathfrak{M}\right) \cap W^{s, p}\left(B^{N}(0,1), \mathfrak{M}\right)$ is not dense in $W^{s, p}\left(B^{N}(0,1), \mathfrak{N}\right)$.

It seems to be quite clear that theorem 2 must be true whenever $s p<M$ but we do not know how to prove that when $0<s<1$. On the other hand we shall see that if $s p>M$ it may be that there is no density even if $s q<M$. We do not know if there is density or not when $s p<M$ and $s q>M$.

In order to prove these density results we need some "stability properties" for the sets $\Lambda_{p q}^{s}\left(\Omega, S^{M}\right)$ with $s \geqslant 1, p \geqslant 1, q \geqslant 1$ under left-composition by Lipschitz functions. It is well known (see [3] for example) that the spaces $W^{m, p}\left(\Omega, \mathbb{R}^{M+1}\right) \cap L^{\infty}\left(\Omega, \mathbb{R}^{M+1}\right)$ are algebras for the pointwise product of functions (for $p=2$ it is the Schauder algebra). This is a simple consequence of the Gagliardo and Nirenberg's inequalities. It turns out that these spaces are also "stable" under left-composition by Lipschitz functions and not only these but also the spaces $\Lambda_{p q}^{s}\left(\Omega, \mathbb{R}^{M+1}\right) \cap L^{\infty}\left(\Omega, \mathbb{R}^{M+1}\right)$. That is to say: for any function $u$ of $\Lambda_{p q}^{s}\left(\Omega, \mathbb{R}^{M+1}\right) \cap L^{\infty}\left(\Omega, \mathbb{R}^{M+1}\right)$ and any function $\Phi$ of $W^{s^{\prime}},{ }^{\infty}\left(\mathbb{R}^{M+1}, \mathbb{R}^{L}\right)$, with $s^{\prime}$ any real greater than $s$ and no less than one, the composed $\Phi_{\text {ou }}$ belongs to $\Lambda_{p q}^{s}\left(\Omega, \mathbb{R}^{L}\right) \cap L^{\infty}\left(\Omega, \mathbb{R}^{L}\right)$. The conditions $s^{\prime} \geqslant 1$ is necessary as shows a result of J. Simon [13].

Acknowledgements. The author wishes to thank Profesor H. Brezis for bringing these problems to his attention. 


\section{PRELIMINARY RESULTS}

First of all let us recall some notations, definitions and well known facts about the classical functional Banach spaces of Besov $\Lambda_{p q}^{s}$ of potential $H^{s, p}$ and of Sobolev $W^{s, p}$. (For all this part see for example $H$. Triebel [14].)

For any non negative integer $m$ and any real $p \geqslant 1$ :

$$
\begin{aligned}
& W^{m, p}\left(\mathbb{R}^{N}\right)=\left\{f \in L^{p}\left(\mathbb{R}^{N}\right) ;\|f\|_{m, p}=\sum_{|\alpha| \leqslant m}\left\|D^{\alpha} f\right\|_{p}<\infty\right\} \\
& W^{o, p}\left(\mathbb{R}^{N}\right)=L^{p}\left(\mathbb{R}^{N}\right) \text { and }\|f\|_{o, p}=\|f\|_{p}
\end{aligned}
$$

For $+\infty>p \geqslant 1, \infty>q \geqslant 1$ and $s>0$, for any integer $M$ greater than $s$ :

$$
\begin{aligned}
& \Lambda_{p q}^{s}\left(\mathbb{R}^{N}\right)=\left\{f \in L^{p}\left(\mathbb{R}^{N}\right) ;\|f\|_{s, p q q}=\|f\|_{p}+\left(\int_{\mathbb{R}^{N}}\left\|\Delta_{h}^{M} f\right\|_{p}^{q} \frac{d h}{|h|^{N+s q}}\right)^{1 / q}<\infty\right\} \\
& \Lambda_{p \infty}^{s}\left(\mathbb{R}^{N}\right)=\left\{f \in L^{p}\left(\mathbb{R}^{N}\right) ;\|f\|\left\|_{s, p ; \infty}=\right\| f \|_{p}^{-}+\sup \left\{\left\{\frac{\left\|\Delta_{h-}^{M} f\right\|_{p}}{|h|^{s}} ; h \in R^{N}, h \neq 0\right\}\right.\right.
\end{aligned}
$$

where

$$
\Delta_{h} f(x)=f\left(x+\frac{h}{2}\right)-f\left(x-\frac{h}{2}\right)
$$

and

$$
\Delta_{h}^{M+1} f=\Delta_{h}\left(\Delta_{h}^{M}\right) \forall M \in \mathbb{N}
$$

In all the following let $\Omega$ be any bounded and smooth domain of $\mathbb{R}^{N}$. One can define the corresponding spaces $W^{m, p}(\Omega), \Lambda_{p q}^{s}(\Omega)$ of functions defined on $\Omega$ as the restriction to $\Omega$ of the functions of the spaces $W^{m, p}\left(\mathbb{R}^{N}\right), \Lambda_{p q}^{s}\left(\mathbb{R}^{N}\right)$. These spaces have an inner description. Namely the space $W^{m, p}(\Omega)$ has the same formal characterisation that $W^{m, p}\left(\mathbb{R}^{N}\right)$, changing $\mathbb{R}^{N}$ by $\Omega$. On the other hand if $0 \leqslant k<s$ and $L>s-k, \forall f \in \Lambda_{p q}^{s}(\Omega)$ :

$$
\|f\|_{s, p ; q}=\|f\|_{p}+\sum_{|x| \leqslant m}\left(\int_{\mathbf{R}^{N}}\left(\int_{\Omega_{h, L}}\left|\Delta_{h}^{L} D^{\alpha} f(x)\right|^{p} d x\right)^{p / q} \frac{d h}{|h|^{N+(s-k-k, q}}\right)
$$

where

$$
\Omega_{h, L}=\bigcap_{j=-L}^{L}\left\{x ; x+j \frac{h}{2} \in \Omega\right\}
$$


When $\Omega$ is a bounded regular domain we have the following continuous embeddings between these different spaces. (See H. Triebel [14], page 195.)

Theorem. i) Let $1 \leqslant p \leqslant \infty, \quad 1 \leqslant p^{\prime} \leqslant \infty, \quad 1 \leqslant q \leqslant \infty, \quad 1 \leqslant q^{\prime} \leqslant \infty$ and $0<s^{\prime}<s<+\infty$. Then $\Lambda_{p q}^{s}(\Omega) \subset \Lambda_{p^{\prime} q^{\prime}(}^{s^{\prime}}(\Omega)$ if $s-N / p>s^{\prime}-N / p^{\prime}$.

ii) Let $1 \leqslant q \leqslant q^{\prime} \leqslant \infty$, and $1 \leqslant p<\infty$ and $0<s<\infty$. Then $\Lambda_{p q}^{s}(\Omega) \subset \Lambda_{p q}^{s}(\Omega)$.

The following rejations between these spaces are well known:

$$
\begin{aligned}
& \text { for } 1 \leqslant p<\infty \text {, and } s>0 \text { non integer } W^{s, p}=\Lambda_{p q}^{s} \\
& \text { for } 1 \leqslant p<\infty \text { and } m \in \mathbb{N} W^{m, p}=H^{m, p} \text {. }
\end{aligned}
$$

Finally we recall a result about extension of functions of $\Lambda_{p q}^{s}(\Omega)$ (respectively $H^{s, p}(\Omega)$ to functions of $\Lambda_{p q}^{s}\left(\mathbb{R}^{N}\right)$ (respectively $H^{s, p}\left(\mathbb{R}^{N}\right)$ ):

Theorem. If $1 \leqslant p \leqslant \infty, 1 \leqslant q \leqslant \infty$ (resp. $1 \leqslant p<\infty, 1 \leqslant q<+\infty)$ and $0<s<$ $+\infty$, then the restriction operator $R$ is a retraction for the space $\Lambda_{p q}^{s}\left(\mathbb{R}^{N}\right)$ (resp. $\left.H^{s, p}(\Omega)\right)$. If $L$ is a natural number then there is a common coretraction from $\Lambda_{p q}^{s}(\Omega)\left(\right.$ resp. $\left.H^{s, p}(\Omega)\right)$ to $\Lambda_{p q}^{s}\left(\mathbb{R}^{N}\right)\left(\right.$ resp. $\left.H^{s, p}\left(\mathbb{R}^{N}\right)\right)$ for all s such that $|s|<L$ for any $1 \leqslant q \leqslant \infty$ and $1 \leqslant p \leqslant \infty$.

Remark 1.1. The Besov spaces can be defined in a more general way for $s \in \mathbb{R}, 0 \leqslant p \leqslant+\infty$ and $0 \leqslant q \leqslant \infty$. These definitions are equivalent to the ones we give here only for $s>0,1 \leqslant p<+\infty$ and $1 \leqslant q \leqslant+\infty$ which are the cases we are interested in. (See [14].)

Let us give now a simple result on the stability of the Sobolev spaces of integer order by left-composition with Lipschitz functions.

Proposition 1.2. Let $p$ be any real number greater or equal than one and $m$ any non negative integer. Consider any vector valued function $\Phi$ belonging to the space $W^{m, \infty}\left(\mathbb{R}^{M}, \mathbb{R}^{L}\right) \cap C^{0}\left(\mathbb{R}^{M}\right)$ such that $\Phi(0)=0$. Then, for any function $u$ of $H^{m, p}\left(\mathbb{R}^{N}, \mathbb{R}^{L}\right) \cap L^{\infty}\left(\mathbb{R}^{N}, \mathbb{R}^{M}\right)$, Фou belongs to $H^{m, p}\left(\mathbb{R}^{N}, \mathbb{R}^{L}\right) \cap L^{\infty}\left(\mathbb{R}^{N}, \mathbb{R}^{L}\right)$ and $\left\|\Phi_{o u}\right\|_{m, p} \leqslant C\|\Phi\|_{m, \infty}\|u\|_{m, p}\left(1+\|u\|_{\infty}^{m-1}\right)$.

Proof. The proof is based on the inequalities of Gagliardo and Nirenberg (see [8]). Let $u$ and $\Phi$ be such as in the hypothesis. We have to estimate the following norm:

$$
\left\|\Phi_{o u}\right\|_{m, p}=\left\|\Phi_{o u}\right\|_{p}+\sum_{j=1}^{N}\left\|D_{j}^{m} \Phi_{o u}\right\|_{p}
$$

By the hypothesis on $\Phi$ it is clear that $\Phi_{o} u$ belongs to $L^{\infty}\left(\mathbb{R}^{N}, \mathbb{R}^{L}\right)$. 
On the other hand, almost everywhere in $\mathbb{R}^{N}$ :

$$
D_{j}^{m}\left(\Phi_{o} u\right)(x)=\sum_{n=1}^{m} \sum_{k_{1}=1}^{M} \ldots \sum_{k_{n}=1}^{M} D_{k_{1} \ldots k_{n}} \Phi(u(x)) \sum_{L_{1}+\ldots+L_{n}=m} a_{L_{1} \ldots L_{2}} D_{j} \iota_{k_{1}}(x) \ldots D_{j} u_{k_{n}}(x)
$$

where the $a_{L_{1} L_{2} \ldots L_{n}}$ are non negative integers depending only of $m$ and $N$.

By Holder's inequality:

$$
\| D_{i}^{m}(\Phi \text { ou })\left\|_{p} \leqslant \sum_{n=1}^{m}\right\| \Phi \|_{n, \infty} \sum_{L_{1}+\cdots+L_{n}=m} a_{L_{1} \ldots L_{n}}\left[\sum_{k_{1}=1}^{M}\left\|D_{i}^{L_{1}} u_{k_{1}}\right\|_{m}^{p}\right] \ldots\left[\sum_{k_{n}=1}^{M}\left\|D_{i}^{L_{n}} u_{k_{n}}\right\|_{m}^{p}\right]
$$

Finally, using the inequalities of Gagliardo and Nirenberg (see [7]) we get:

$$
\left\|D_{i}^{m}(\Phi o u)\right\|_{p} \leqslant C(N, m)\|\Phi\|_{m, \infty}\|u\|_{m, p}\left(1+\|u\|_{\infty}^{m-1}\right)
$$

Remark 1.3. By the same method and using the dominated convergence theorem one can prove the following: If $\left\{u^{n}\right\}$ is a sequence of functions of $H^{m, p}\left(\mathbb{R}^{N}, \mathbb{R}^{M}\right) \cap L^{\infty}\left(\mathbb{R}^{N}, \mathbb{R}^{M}\right)$ such that:

i) $u^{n} \rightarrow u$ in $H^{m, p}\left(\mathbb{R}^{N}, \mathbb{R}^{M}\right)$.

ii) $\exists C>0 ;\left\|u^{n}\right\|_{\infty} \leqslant C \forall n \in \mathbb{N}$ and $\|u\|_{\infty} \leqslant C$.

iii) $\Phi \in C^{m}\left(\mathbb{R}^{M}, \mathbb{R}^{L}\right)$.

then there is a subsequence of $\left\{\Phi o u^{n}\right\}$ converging to $\Phi$ ou in $H^{m, p}\left(\mathbb{R}^{N}, \mathbb{R}^{L}\right)$.

Remark 1.4. Proposition 1.2 and remark 1.3 remain true if we consider $u$ belonging to $W^{m, p}\left(\Omega_{1}, \mathbb{R}^{M}\right)$ and $\Phi$ of $W^{m, \infty}\left(\Omega_{2}, \mathbb{R}^{L}\right)$ where $\Omega_{1}, \Omega_{2}$ are regular domains of $\mathbb{R}^{N}$ and $\mathbb{R}^{L}$ respectively and $\operatorname{Im}(t) \subset \Omega_{2}$. If $\Omega_{1}$ is bounded the condition $\Phi(0)=0$ is not necessary (this condition is only needed in order to prove that $\Phi$ ou belongs to $L^{p}$ but if $\Omega$ is bounded that is true as soon as $\Phi o u$ belongs to $L^{\infty}$ ).

In order to extend this simple result to more general spaces we shall need the following lemma.

Lemma 1.5. Let $s$ be any non negative real. Then for any $1 \leqslant p<\infty$ and $1 \leqslant q \leqslant \infty$ :

$$
\Lambda_{p q}^{s} \cap L^{\infty} \subset \Lambda_{r p, r q}^{s i r} \quad \forall r \geqslant 1
$$

and

$$
\forall u \in \Lambda_{p q}^{s} \cap L^{\infty}:\|u\|_{s ; r, r p, r q} \leqslant C(s, r)|| u\left\|_{s, p ; q}^{1 / r}\right\| u \|_{\infty}^{1-1 / r}
$$


Proof. Let $M$ be any integer such that $M>s \geqslant s / r$ for any $r \geqslant 1$. Then

$$
\begin{gathered}
u \in \Lambda_{p q}^{s} \Leftrightarrow\|u\|_{p}+\left\{\int \frac{\left\|\Delta_{h}^{M} u\right\|_{q}^{p}}{|h|^{N+s q}} d h\right\}^{\frac{1}{q}}<\infty \\
u \in \Lambda_{r p, r q}^{s / r} \Leftrightarrow\|u\|_{r p}+\left\{\int \frac{\left\|\Delta_{h}^{M} u\right\|_{r p}^{r q}}{|h|^{N+s q}} d h\right\}^{\frac{1}{r^{q}}}<\infty
\end{gathered}
$$

but:

$$
\left\|\Delta_{h}^{M} u\right\|_{r p}^{r q}=\left\{\int_{\mathbf{R}^{N}}\left|\Delta_{h}^{M} u(x)\right|^{r p} d x\right\}^{i \frac{q}{p}} \leqslant\left((M+1)\|u\|_{\infty}^{r-1}\right)^{q}\left\{\int_{\mathbf{R}^{N}}\left|\Delta_{h}^{M} u(x)\right|^{p} d x\right\}^{q}
$$

and that gives the inclusion and the inequality for $q<\infty$. For $q=\infty$ the proof is similar.

We can prove now the following:

Proposition 1.6. Let $s$ be any non negative real, $1 \leqslant p<\infty, 1 \leqslant q \leqslant \infty$ and $m$ the integer part of $s$. Consider any vector valued function $\Phi$ of $W^{s^{\prime}, \infty}\left(\mathbb{R}^{M}, \mathbb{R}^{L}\right) \cap C^{0}, \max (1, s)<s^{\prime}<m+1$, such that $\Phi(0)=0$. Then for any function $u$ of the space $\Lambda_{p q}^{s}\left(\mathbb{R}^{N}, \mathbb{R}^{M}\right) \cap L^{\infty}\left(\mathbb{R}^{N}, \mathbb{R}^{M}\right)$ the function $\Phi$ ou belongs to $\Lambda_{p q}^{s}\left(\mathbb{R}^{N}, \mathbb{R}^{L}\right) \cap L^{\infty}\left(\mathbb{R}^{N}, \mathbb{R}^{L}\right)$ and $\left\|\Phi_{o u}\right\|_{s, p ; q} \leqslant C\|\Phi\|\left\|_{s^{\prime}, \infty}\right\| u \|_{s, p ; q}\left(\|u\|_{\infty}^{m}+1\right)$.

Proof. We shall consider on the Besov space $\Lambda_{p q}^{s}\left(\mathbb{R}^{N}, \mathbb{R}^{M}\right)$ the norm

$$
\|u\|_{s, p ; q}=\|u\|_{p}+\left\{\int \frac{\left\|\Delta_{y}^{L} u\right\|_{q}^{p}}{|y|^{N+s q} d y}\right\}^{\frac{1}{q}} \quad \text { where } \quad L=m+1
$$

As $\Phi(0)=0$ and $\Phi$ is Lipschitz it is clear that $\Phi$ ou belongs to $L^{p}$. On the other hand it is simple (but tedious) to see by induction on $m$ that:

$$
\begin{gathered}
\Delta_{y}^{m} \Phi_{o} u=\sum_{j=0}^{m-1} \sum_{r=0}^{j} \sum_{k_{1}+\ldots+k_{r}=j} \int_{0}^{1} \ldots \int_{0}^{1} b_{k_{1} \ldots k_{r j} j} d^{r+1} \Phi\left(G_{-y \cdot(m-r-1)} \delta_{y s_{1}} \ldots \delta_{y s_{r+1}} u\right) \\
\left.G_{y \cdot j} \Delta_{y}^{m-j} u G_{-y \cdot\left(m+k_{1}-2 j-1\right)} \Delta_{y}^{k_{1}} \delta_{y s_{1}} u \ldots G_{-y \cdot\left(m+2 k_{1}+\ldots+2 k_{i}+k_{i+1}-2 j-i+1\right)}\right) \Delta_{y^{\prime}+1}^{k_{i}} \delta_{y s_{1}} \ldots \\
\ldots \delta_{y s_{1}+1} u \ldots G_{-y \cdot\left(m+2 k_{1}+\ldots+2 k_{r-1}+k_{r}-2 j-r\right)} \Delta_{y}^{k_{2}} \delta_{y s_{1}} \ldots \delta_{y s_{r}} u d s_{1} \ldots d s_{r+1}
\end{gathered}
$$

where:

$$
b_{k_{1} \ldots k_{j} j}=\left[\begin{array}{c}
m-1 \\
m-j-1
\end{array}\right] \cdot\left[\begin{array}{c}
j-1 \\
k_{1}-1
\end{array}\right] \cdot\left[\begin{array}{c}
j-k_{1}-1 \\
k_{2}-1
\end{array}\right] \cdot\left[\begin{array}{c}
j-k_{1}-k_{2}-\ldots-k_{r-1}-1 \\
k_{r}-1
\end{array}\right]
$$

for any $r \in[0, j], j \in[0, m-1]$ 
with:

$$
\left[\begin{array}{l}
a \\
b
\end{array}\right]=\frac{a !}{b !(a-b) !}
$$

$\delta_{y s} u(x)=s u(x+y / 2)+(1-s) u(x-y / 2)$ for any $s \in(0,1), x \in \mathbb{R}^{N}, y \in \mathbb{R}^{N}$ and $G_{y} u(x)=u(x+y / 2)$.

By this:

$$
\Delta_{y}^{m+1} \Phi_{o u}=\Delta_{y}\left(\Delta_{y}^{m} \Phi_{o u}\right)=A_{1}+A_{2}+A_{3}
$$

where:

$$
\begin{gathered}
A_{1}=\sum_{j=0}^{m-1} \sum_{r=0}^{j} \sum_{k_{3}+\ldots+k_{r}=j} b_{k_{1} \ldots k_{r} j} \int_{0}^{1} \ldots \int_{0}^{1} d^{r+1} \Phi\left(G_{-y \cdot(m-r-1)} \delta_{y s_{1}} \ldots \delta_{y s_{r+1}} u\right) G_{y \cdot(j+1)} \Delta_{y}^{m-j} u \\
\ldots G_{-y \cdot\left(m+2 k_{1}+\ldots+2 k_{r-1}-2 j-r+1\right)} \Delta_{y}^{k} \delta_{y s_{1}} \ldots \delta_{y s_{r}} u d s_{1} \ldots d s_{r+1} \\
A_{2}=\sum_{j=0}^{m-1} \sum_{r=0}^{j} \sum_{k_{1}+\ldots k_{r}=j} b_{k_{1} \ldots k_{r} j} \int_{0}^{1} \ldots \int_{0}^{1} d^{r+1} \Phi\left(G_{-y \cdot(m-r-1)} \delta_{y s_{1}} \ldots \delta_{y s_{r}+1} u\right) G_{y \cdot j} \Delta_{y}^{m-j+1} u \\
G_{-y \cdot\left(m+k_{2}-2\right)} \Delta_{y}^{k_{1}} \delta_{y s_{1}} u \ldots G_{-y \cdot\left(m-k_{r}-r+1\right)} \Delta_{y}^{k_{r}} \delta_{y s_{1}} \ldots \delta_{y s_{r}} u d s_{1} \ldots d s_{r+1} \\
A_{3}=\sum_{j=0}^{m-1} \sum_{r=0}^{j} \sum_{k_{1}+\ldots+k_{r}=j} b_{k_{3} \ldots k_{r} j} \int_{0}^{1} \ldots \int_{0}^{1} \Delta_{y}\left(d^{r+1} \Phi\left(G_{-y \cdot(m-r-1)} \delta_{y s_{1}} \ldots \delta_{y s_{r+1}} u\right)\right) \\
G_{y(j-1)} \Delta_{y}^{m-j} u \ldots G_{-y\left(m-k_{r}-r+1\right)} \Delta_{y}^{k_{r}} \delta_{y s_{1}} \ldots \delta_{y s_{r}} u d s_{1} \ldots d s_{r+1}
\end{gathered}
$$

Using Holder's inequalities, the translation invariance of $L^{p}$ norms and lemma 1.5 one gets:

$$
\left\|A_{i}\right\|_{s, p ; q} \leqslant C\|\Phi\|_{m, \infty}\|u\|_{s, p ; q} \sum_{r=0}^{m}\|u\|_{\infty}^{r} \quad \text { for } \quad i=1,2
$$

In order to obtain the same estimate for $A_{3}$ we have to use also that, by the hypothesis on $\Phi$ :

$$
\begin{gathered}
\forall \xi, \zeta \in \mathbb{R}^{M}, \forall r \leqslant m-1, \forall v_{i} \in \mathbb{R}^{M} i=1, \ldots, r+1: \\
\left|\left[d^{r+1} \Phi(\xi)-d^{r+1} \Phi(\zeta)\right] v_{1} \ldots v_{r+1}\right| \leqslant C|| \Phi \|_{s^{\prime}, \infty}|\xi-\zeta|^{s^{\prime}-m}\left|v_{1}\right| \ldots\left|v_{r+1}\right|
\end{gathered}
$$


Remark 1.7. One can prove a result similar to the one of Remark 1.3 for the spaces $\Lambda_{p q}^{s}\left(\mathbb{R}^{N}, \mathbb{R}^{M}\right) \cap L^{\infty}\left(\mathbb{R}^{N}, \mathbb{R}^{M}\right)$.

Remark 1.8. Proposition (1.6) remains true if we consider $u$ belonging to the space $\Lambda_{p q}^{s}\left(\Omega_{1}, \mathbb{R}^{M}\right) \cap L^{\infty}\left(\Omega_{1}, \mathbb{R}^{M}\right)$ of functions defined on a smooth domain of $\mathbb{R}^{N}$ and $\Phi$ a function of $W^{s^{\prime}, \infty}\left(\Omega_{2}, \mathbb{R}^{\prime}\right)$ where $\Omega_{2}$ is a smooth domain of $\mathbb{R}^{M}$ containing $/ m(u)$. Just using the extension operators from $\Lambda_{p q}^{s}\left(\Omega_{1}, \mathbb{R}^{M}\right)$ to $\Lambda_{p q}^{s}\left(\mathbb{R}^{N}, \mathbb{R}^{M}\right)$, from $W^{s^{\prime}, \infty}\left(\Omega_{2}, \mathbb{R}^{L}\right)$ to $W^{s^{\prime}, \infty}\left(\mathbb{R}^{M}, \mathbb{R}^{L}\right)$ and their inverse.

As in remark (1.4) if $\Omega_{1}$ is bounded we do not need no more the condition $\Phi(0)=0$ in order to have the results of proposition (1.6).

Remark 1.9. The condition $s^{\prime} \geqslant 1$ is necessary as shows the following result of J. Simon [13]:

Theorem. (J. Simon). Given $p \in(0,1), \forall s \in(0,1)$ and $\forall \varepsilon>0$, $\exists w \in W^{s, r}(\Omega) \forall r \in[1, \infty] ;|w|^{p-1} w \notin W^{s p+\varepsilon, r^{\prime}}(\Omega) \forall r^{\prime} \in[1, \infty]$.

In fact, J. Simon gives a counterexample where the function $w$ is Lipschitz of order $s$ on $\Omega$.

Remark 1.10. It is well known that for any non negative integer $m$ the space $W^{m, p} \cap L^{\omega \nu}$ is an algebra (for $p=2$ it is the Schauder algebra). The proof is a simple consequence of the formula of Leibnitz and the Gagliardo and Nirenberg's inequalities.

This result remains true for any Besov space $\Lambda_{p q}^{s}$ with $s>0, p \geqslant 1$ and $q \geqslant 1$. We only have to show this for the homogeneous spaces $\dot{\Lambda}_{p q}^{s}$. But this is very easy using the characterisation of these spaces given by $J$. Dorronsoro in [6]: $u \in \Lambda_{p q}^{s}$ iff there is an integer $M>s$ such that, if $Q$ is any cube in $\mathbb{R}^{N}$ and $P_{Q}^{M}(f)$ is the unique polynomial in $\mathbf{P}_{M}$ (the space of polynomials of degree less or equal than $M$ ) such that:

$$
\int_{Q}\left(f-P_{Q}^{M}(f)\right) x^{x} d x=0 \quad \forall \alpha \in \mathbb{N}^{N} ;|\alpha|<M
$$

and if

$$
\Omega_{f, M}(x, t)=\sup \left\{|Q|^{-1} \int_{Q}\left|f-P_{Q}^{M}(f)\right| d z ; x \in Q|Q|=t^{N}\right\}
$$

one has:

$$
\left(\int_{Q}\left(t^{-x}\left\|\Omega_{f, M}(., t)\right\|_{p}\right)^{q_{t}-1} d t\right)^{\frac{1}{q}<\infty}
$$


and the fact that $P_{Q}^{M}$ satisfies:

$$
\left\|P_{Q}^{M}(v)\right\|_{\infty} \leqslant C|Q|^{-1} \cdot \int_{Q}|v| d z \leqslant C\|v\|_{\infty} \quad \text { (see [6]) }
$$

The same result is true for the space $H^{s, p} \cap L^{\infty}$ using similar results of $\mathrm{J}$. Dorronsoro about Bessel-potential spaces (see [7]). The author is grateful to J. R. Dorronsoro for fruitful conversations about this Remark.

\section{DENSITY RESULTS}

Let $\Omega$ be a bounded and regular domain of $\mathbb{R}^{N}$ and $S^{M}$ the unit sphere of $\mathbb{R}^{M+1}$. As it has been said in the introduction we prove in this section the density of the set of regular functions defined on $\Omega$ and taking values on $S^{M}$, on the sets $\Lambda_{p q}^{s}\left(\Omega, S^{M}\right)$, when some relations hold between the coeficients $s, p, q, M$, and $N$.

First we give a simple extension of a density result in [2] and [12]:

Theorem 2.1. Let $M, N$ and $m$ three positive integers and $p$ a real no less than one such that $m p \geqslant N$. Then $C\left(\Omega, S^{M}\right) \cap W^{m, p}\left(\Omega, S^{M}\right)$ is dense in $W^{m, p}\left(\Omega, S^{M}\right)$.

Proof. Any function $u$ of $W^{m, p}\left(\Omega, S^{M}\right)$ is bounded and then belongs to $W^{1, m p}\left(\Omega, S^{M}\right)$ by the Gagliardo and Nirenberg's inequalities. Now using the method of [2] we obtain, by taking averages of $u$ over balls, a sequence of continuous functions $\left\{u_{\varepsilon}\right\}$ converging to $u$ in $W^{1, m p}\left(\Omega, S^{M}\right)$ when $\varepsilon$ tends to zero and such that $\operatorname{dist}\left(u_{\varepsilon}(x), S^{M}\right)$ tends to zero uniformly on $\Omega$ with $\varepsilon$ (using that $m p \geqslant N$ ). The result follows as in [2].

Theorem 2.2. Let $M$ and $N$ be two positive integers. For $p$ and $q$ reals greater than one and any non negative real $s$ such that $s p \geqslant N$, the set $C\left(\Omega, S^{M}\right) \cap \Lambda_{p q}^{s}\left(\Omega, S^{M}\right)$ is dense in $\Lambda_{p q}^{s}\left(\Omega, S^{M}\right)$.

Proof. Here again the idea of the proof is the same as in [2].

Consider any function $u$ of $\Lambda_{p q}^{s}\left(\Omega, S^{M}\right)$ and its extension to $\mathbb{R}^{N}, U$ of $\Lambda_{p q}^{s}\left(\mathbb{R}^{N}, \mathbb{R}^{M+1}\right)$. Define for any $\varepsilon>0$;

$$
U_{\varepsilon}(x)=\left|B_{\varepsilon}(x)\right|^{-1} \int_{B_{\varepsilon}(x)} U(z) d z \quad \text { where } \quad B_{\varepsilon}(x)=\left\{z \in \mathbb{R}^{N} ;|z-x| \leqslant \varepsilon\right\}
$$

These functions belong to $C\left(\mathbb{R}^{N}, S^{N}\right)$ and if $u_{\varepsilon}=U_{\varepsilon \downarrow s}$ then, as $\varepsilon \rightarrow 0, u_{\varepsilon} \rightarrow u$ in $\Lambda_{p q}^{s}\left(\Omega, \mathbb{R}^{M+1}\right)$. On the other hand consider any real $r \geqslant 1$ such that $1 / p<s / r \leqslant 1$. For any $x \in \Omega$

$$
\left[\operatorname{dist}\left(u_{\varepsilon}(x), S^{M}\right)\right]^{\frac{s p}{r}} \leqslant\left|B_{\varepsilon}\right|^{-1} \int_{B_{s}(x)}\left|U(y)-u_{\varepsilon}(x)\right|^{\frac{s p}{r}} d y \leqslant C \int_{B_{z \varepsilon}(0)} \int_{B_{\varepsilon}(x)} \frac{|U(z)-U(z-h)|^{\frac{s p}{r}}}{\left|\nabla_{\varepsilon}\right|^{2}} d z d h
$$


Now if $q>p$ :

$$
\begin{gathered}
C p: \quad \iint_{B_{2 \varepsilon}(0)} \frac{|U(z)-U(z-h)|^{\frac{s p}{r}}}{\mid B_{\varepsilon}(x)} d z d h \leqslant \\
C\left\{\int\left(\int_{B_{2 \varepsilon}(0)}\left(\int_{B_{\varepsilon}(x)}|U(z)-U(z-h)|^{\frac{s p}{r}} d z\right)^{\frac{q}{p}} \frac{d h}{|h|^{\overline{N+} s q}}\right\}^{\frac{p}{q}}\right.
\end{gathered}
$$

using that $s p \geqslant N$.

Therefore [dist $\left.\left(u_{\varepsilon}(x), S^{M}\right)\right]^{s p / r} \rightarrow O$ when $\varepsilon \rightarrow \infty$ uniformly for $x \in \Omega$. If $q \leqslant p, \Lambda_{p q}^{s} \subset \Lambda_{p p}^{s}$ and then:

$$
\iint_{\mathbb{R}^{N}}\left[|U(z)-U(y)|^{s p / r} /|z-y|^{N+s p}\right] d y d z<\infty
$$

Using again that:

$$
\left[\operatorname{dist}\left(u_{\varepsilon}(x), S^{M}\right)\right]^{s p / r} \leqslant \int_{B_{\varepsilon}} \int_{B_{\varepsilon}}\left[|U(z)-U(y)|^{s p / r} /|z-y|^{N+s p}\right] d y d z
$$

we get the same conclusion.

Taking now $v_{\varepsilon}=\operatorname{Projs}^{2} u_{\varepsilon}$ for $\varepsilon$ small enough we obtain the result.

So we shall consider in all the following that $s p<N$. In this case our first purpose is to approximate any function of $\Lambda_{p q}^{s}\left(\Omega, S^{M}\right)$ by functions, not necessarily continuous taking values only on a segment of sphere. In order to do this we shall need the following deformation lemma.

Lemma 2.3. For any $\varepsilon>0$ and $x^{0} \in S^{M}$ let $V_{x^{0}, \varepsilon / 2}=S^{M} \cap B^{M+1}\left(x^{0}, \varepsilon / 2\right)$ and $W_{x^{0}, \varepsilon / 2}=S^{M}-\operatorname{Int}\left(V_{x^{0}, \varepsilon / 2}\right)$. There is a $C^{\infty}$ map $\Phi_{\varepsilon}$ from $S^{M}$ to $W_{x^{0}, \varepsilon / 2}$ such that:

i) $\Phi_{k \mid W_{x^{0}, \varepsilon / 2}}=I d_{\mid W_{x^{0}, k / 2}}$.

ii) $\forall L \in \mathbb{N}, \exists C>0 ; \forall \alpha \in \mathbb{N}^{M+1}|\alpha| \leqslant L ;\left\|D^{\alpha} \Phi\right\|_{\infty} \leqslant \mathrm{CE}^{-|\alpha|}$.

Proof. This is a "regular version» of a lemma proved in [5] (where $\Phi$ has only to be Lipschitz). The proof is essentialy the same.

We can prove now our first density result for the particular case of Sobolev spaces $W^{m, p}\left(\Omega, S^{\mathrm{w}}\right)$ with $m$ an integer.

Theorem 2.4. For any real $p$, greater or equal than one, for any positive integer $M$ and any non negative integer $m$ such that $m p<M$ the space $C^{\infty}\left(\Omega, S^{M}\right)$ is dense in $W^{m, p}\left(\Omega, S^{M}\right)$. 
Proof. Let $\varepsilon>0$ be fixed. By lemma 2.1, for any $x^{0} \in S^{M}$ there is a $C^{\infty}$ function $\Phi_{\varepsilon}$ from $S^{M}$ to $W_{x^{0}, z / 2}$ such that:

i) $\Phi_{\varepsilon \mid W_{x^{0}, \varepsilon / 2}}=I d_{\mid W_{r}, \varepsilon / 2}$.

ii) $\forall L \in \mathbb{N}, \exists C>0 ; \forall \alpha \in \mathbb{N}^{M+1}|\alpha| \leqslant L ;\left\|D^{\alpha} \Phi_{\text {; }}\right\|_{\infty} \leqslant C \varepsilon^{-|x|}$.

In order to apply the results of section 1 we extend this function to all of $\mathbb{R}^{M+1}$. For this purpose consider any $C^{\infty}$ function $h$ from $\mathbb{R}^{+}$to $\mathbb{R}^{+}$such that $h(1)=1$, supp $h \subset(1 / 2,3 / 2)$ and define $\Psi_{x^{0}, \varepsilon}=\Phi_{x^{0}, \mathrm{e}}(x /|x|) \cdot h(x)$. This function is $C^{\infty}$ and $D^{\alpha} \Psi(x) \leqslant C \cdot \varepsilon^{-|\alpha|}$ for any $x$ of $\mathbb{R}^{M+1}$ and $\alpha$ such that $|\alpha| \leqslant L$.

If $P_{\varepsilon}$ is the maximal number of disjoints sets of the form $V_{x^{0}, \varepsilon}$ contained in $S^{M}$ there is a constant $K$ such that $P_{\varepsilon} \geqslant K \cdot \varepsilon^{-M}$. Let $\left\{V_{x^{\prime},{ }_{1}}\right\}_{i=1, \ldots . .} P_{\varepsilon}$ such a family of sets and define

$$
\forall i \in\left\{1, \ldots, P_{\varepsilon}\right\} \quad u_{i, s}=\Psi_{x^{\prime}, c o u} \equiv \Phi_{x^{\prime}, \tilde{a}} \text { ou }
$$

By construction $u_{i, \varepsilon}=u$ on $W_{x^{\prime}, s / 2}$ and $u_{i, \varepsilon}$ goes from $\Omega$ to $W_{x^{\prime}, \varepsilon / 2}$.

On the other hand:

$$
\begin{aligned}
& \sum_{i=1}^{P_{i}}\left\|u-u_{i, \varepsilon}\right\|_{m, p}^{p_{m}}=\left[\left\|\sum_{i=1}^{P_{e}}\left(u-u_{i, \varepsilon}\right)\right\|_{m, p}\right]^{p}= \\
& =\left[\left\|\sum_{i=1}^{P_{e}}\left(I d-\Psi_{i, \varepsilon}\right) o u\right\|_{m, p}\right]^{p} \leqslant C\left\|\Phi_{\varepsilon} o u\right\|_{m, p}^{p}
\end{aligned}
$$

with $\Phi_{s}(x)=\Sigma\left(x-\Psi_{i, c}(x)\right)$ for any $x$ of $\mathbb{R}^{M+1}$. $\Phi_{b}$ is. a $C^{\infty}$ function such that $\Phi_{b}(0)=0$ and then by proposition (1.2):

$$
\sum_{i=1}^{p_{t}}|| u-\left.u_{i, \varepsilon}\right|_{m, p} ^{p} \leqslant C\left\|\mid \Phi_{\varepsilon}\right\|_{m, \infty}^{p}\|u\|_{m, p}^{p}
$$

But, by definition of $\Phi_{\varepsilon}$ and using that the supports of the functions $\left(I d-\Psi_{i, \varepsilon}\right)$ and $\left(I d-\Psi_{j, \varepsilon}\right)$ are disjoints for $i \neq j$ we have:

$$
\left\|\Phi_{\varepsilon}\right\|_{m, \infty} \leqslant \max \left\{\left\|I d-\Psi_{i, \varepsilon}\right\|_{m, \infty} ; i=1, \ldots, P_{\varepsilon}\right\} \leqslant C \varepsilon^{-m}
$$

from this we deduce:

$$
\sum_{i=1}^{P_{z}}\left\|u-u_{i, \varepsilon}\right\|_{m, p}^{p} \leqslant C \varepsilon^{-i n p}|| u \|_{m, p}^{p}
$$

It follows that there is at least one $i \in\left\{1, \ldots, P_{\varepsilon}\right\}$ such that:

$$
\left\|u-u_{i, \varepsilon}\right\|_{m, p}^{p} \leqslant C \varepsilon^{-m p}\left(P_{\varepsilon}\right)^{-1} \cdot\|u\|_{n, p}^{p} \leqslant C \varepsilon^{M-m p} \cdot\|u\|_{m, p}^{p}
$$


This inequality gives us an approximation of any function $u$ of $W^{m, p}$ taking values on $S^{M}$ by functions $u_{i, \varepsilon}$ of $W^{m, p}$ with values only in a segment of sphere $W_{x^{i}, \varepsilon}$. We have to approximate now $u_{i, a}$ by a smooth map from $\Omega$ to $S^{M}$. There is no loss of generality if we suppose that $x^{i}=(1,0, \ldots, 0)$. Consider the stereographic projection $\mathfrak{P}$ of $S^{M}$ on $\mathbb{R}^{M}$ with pole $x^{i}$. Its restriction $P$ to $W_{x^{\prime}, \varepsilon}$ is a $C^{\infty}$ diffeomorphism from $W_{x^{\prime}, \varepsilon}$ into $P\left(W_{x^{\prime}, \varepsilon}\right)$.

Let us define now: $\forall z \in B^{M+1}(0,1) \mid B^{M+1}\left(x^{i}, \varepsilon\right), \quad z \neq 0 ; \quad P^{E}(z)=h(|z|) \cdot P(z /|z|)$ and $P^{E}(0)=0$ (where $h$ is as in 2.4). It is a $C^{\infty}$ extension of $P$ to $B^{M+1}(0,1) /$ $B^{M+1}\left(x^{i}, \varepsilon\right)$. On the other hand the image of $\left[B^{M+1}(0,1)\right)\left(B^{M+1}\left(x^{i}, \varepsilon\right)\right]$ by $P^{E}$ is
contained in $P\left(W_{x^{\prime}}\right)$.

By the results of section $1 P^{F}\left(u_{i, \varepsilon}\right)$ is now a function of $W^{m, p}\left(\Omega, \mathbb{R}^{M}\right) \cap L^{\infty}\left(\Omega, \mathbb{R}^{M}\right)$ and can be approximated by smooth functions $v_{i, c, n}$ of $C^{\infty}\left(\Omega, \mathbb{R}^{M}\right) \cap L^{\infty}\left(\Omega, \mathbb{R}^{M}\right)$. Define now $u_{i, c, n}=P^{-1}\left(v_{i, r, n}\right)$. These functions belongs to $C^{\infty}\left(\Omega, \mathbb{R}^{M}\right)$. We want to show that there is a subsequence tending to $u_{i, \varepsilon}$ in $W^{m, p}$. By remark 1.3 this holds because:

i) $v_{i, c . n} \rightarrow P^{E}\left(u_{i, \varepsilon}\right)$ in $W^{m, p}\left(\Omega, \mathbb{R}^{M}\right)$.

ii) $\exists C>0 ; \forall n\left\|v_{i, r, n}\right\|_{\infty} \leqslant C$.

and then $u_{i, \varepsilon, k} \rightarrow P^{-1}\left(P^{E}\left(u_{i, n}\right)\right) \equiv u_{i, x}$ in $W^{m, p}$ for a subsequence of $u_{i, \varepsilon, n}$.

In order to prove a similar result for the space $\Lambda_{p q}^{s}$ we would like to apply the same method. Unfortunately formula (2.5) is not true for these spaces. Nevertheless one can, with a slight modification prove the following:

Theorem 2.6. For any bounded smooth domain $\Omega$ of $\mathbb{R}^{N}$ and three reals $1 \leqslant p<\infty, 1 \leqslant q \leqslant \infty, s \geqslant 1$ such that $\operatorname{s.max}(p, q)<M$, the space $C^{\infty}\left(\Omega, S^{m}\right)$ is dense in $\Lambda_{p q}^{s}\left(\Omega, S^{M}\right)$.

Proof. As in 2.4 consider, for any $\varepsilon>0$ fixed, a family $\left\{V_{x^{i}, \varepsilon}\right\}_{i=1, \ldots, p_{x}}$ of disjoints subsets of $S^{M}$ such that $P_{\varepsilon} \geqslant K \varepsilon^{-M}$ and $\Phi_{x^{\prime}, c}$ the corresponding function from $S^{M}$ to $W_{x^{i}, \varepsilon / 2}$ equals to the identity on $W_{x^{\prime}, \varepsilon / 2}$. Define now $\Psi_{x^{\prime}, \varepsilon}(x)$ $=h(|x|) \cdot \Phi_{x^{i}, \varepsilon}(x /|x|), \quad \Psi_{x^{i}, \varepsilon}(0)=0$ where $h$ is as in 2.4 . Let $U$ be the image of $u$ by the extension operator from $\Lambda_{p q}^{s}\left(\Omega, S^{M}\right)$ to $\Lambda_{p q}^{s}\left(\mathbb{R}^{N}, \mathbb{R}^{M+1}\right)$. Define $U_{i, s}=\Psi_{x^{\prime}, q} U$ and consider the function $D_{i, s}=F_{i, o} U$ where we have posed
$F_{i, s}(z)=h(|z|) \cdot z /|z|-\Psi_{x^{i}, \varepsilon}(z)$.

Observe first of all that

$$
\operatorname{supp} D_{i, \varepsilon} \subset Q_{i, \varepsilon}=\left\{x \in \mathbb{R}^{N} ; U(x) /|U(x)| \in V_{x^{i, \varepsilon}}\right\} \text { and } Q_{i, \varepsilon} \cap Q_{j, \varepsilon}=\phi \text { if } i \neq j
$$

and therefore for any integer $L$ :

$$
\operatorname{supp} \Delta_{y}^{L} D_{i, \varepsilon} \subset \bigcup_{k=-L}^{k=+L} \tau_{y k} Q_{i, k} \equiv A^{i, \varepsilon}(y)
$$


with:

$$
\tau_{y k} Q_{i, \varepsilon}=\left\{x \in \mathbb{R}^{N} ; x+y k \in Q_{i, \varepsilon}\right\}
$$

Let be $m$ the integer part of $s$; arguing as in (1.7), for any $i \in\left\{1, \ldots, P_{e}\right\}$ :

$$
\Delta_{y}^{m+1} D_{i, \varepsilon}=S_{1, y}^{i, \varepsilon}+S_{2, y}^{i, \varepsilon}+S_{3, y}^{i, \varepsilon}
$$

Now we have to estimate the sum $\sum_{i=1}^{P_{\varepsilon}}\left\|D_{i, \varepsilon}\right\|_{s ; \text {; }}^{q}$. The way to do this is the same as for $A_{i}(i=1,2,3)$ in $(1.7)$ with a slight modification. As the sets $A^{i, t}(y)$ are not translation invariants we have to use the properties of the supports of the functions $D_{i, \varepsilon}$ in the following way:

$$
\begin{aligned}
& \sum_{i=1}^{\mathbf{P}_{\ell}} \int_{\mathbf{R}^{n}}\left\|G_{y r} \Delta_{y}^{L} U\right\|_{L^{i}\left(A^{i} i^{i(y)}(y)\right)} \frac{d y}{|y|^{N+s q}} \leqslant
\end{aligned}
$$

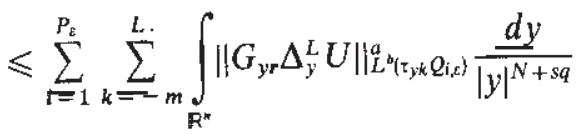

$$
\begin{aligned}
& \leqslant\left[P_{\varepsilon}\right]^{\left(1-\frac{a}{b}\right)^{+}} C(L) \int_{\mathbf{D}^{n}}\left\|G_{y r} \Delta_{y}^{L} U\right\|_{L^{b}\left(\mathbb{R}^{N}\right)}^{a} \frac{d y}{|y|^{N}+s q}
\end{aligned}
$$

for any integers $r$ and $L$ and two any finite reals $a \geqslant 1, b \geqslant 1$.

And we obtain for $s^{\prime}>s$ :

$$
\left(\sum_{i=1}^{P_{\varepsilon}}\left\|D_{i, \varepsilon}\right\|_{s, p ; q}^{q}\right)^{1 / q} \leqslant C \varepsilon^{-s^{\prime}} P^{\left(\frac{1}{q}-\frac{1}{p}\right)^{+}}\|U\|_{s, p ; q}
$$

We choose $s^{\prime}$ such that $s^{\prime} \max (p, q)<M$.

From (2.9) we deduce that, for at least one $i$ of $\left\{1, \ldots, P_{\varepsilon}\right\}$ we have:

$$
\left\|D_{i, s}\right\|_{s, p ; q} \leqslant C \varepsilon^{-s^{\prime}}\left[P_{\varepsilon}\right]^{-\min (1 / p, 1 / q)}\|U\|_{s, p: q} \leqslant C \varepsilon^{-s^{\prime}+M \min (1 / p, 1 / q)}\|U\|_{s, p: q}
$$

Now call $u_{i, \varepsilon}=U_{i, \varepsilon[2 .}$. Then $u_{i, \varepsilon} \in \Lambda_{p q}^{s}\left(\Omega, W_{x^{\prime}, \varepsilon}\right)$ and $D_{i, \varepsilon}$ is an extension of $u-u_{i, \varepsilon}$ to all of $\mathrm{P}^{N}$. Therefore:

$$
\left\|u-u_{i, \varepsilon}\right\|_{s, p ; q} \leqslant\left\|D_{i, \varepsilon}\right\|_{s, p ; q} \leqslant C_{\varepsilon}^{\left[M \min (t / p, 1 / 4)-s^{\prime}\right]}|| u \|_{s, p ; q}
$$

The proof now follows as above using stereographic projection.

Remark 2.10. If $s p>M$, the conclusion can fail even if $s q<M$. For example, it is known (see [2], [5] and [12]) that the function $x /|x|$ belongs to 
$H^{1}\left(B^{3}, S^{2}\right)$ and can not be approximated by functions of $C^{\infty}\left(B^{3}, S^{2}\right)$ in $H^{1}\left(B^{3}, S^{2}\right)$. On the other hand $x /|x|$ belongs to $W^{2, r}\left(B^{3}, S^{2}\right)$ for any $r<3 / 2$.

Now, using the inclusion properties recalled in the first section:

$W^{2, r}\left(B^{3}, S^{2}\right) \subset \Lambda_{r 1}^{2-\varepsilon}\left(B^{3}, S^{2}\right) \forall \varepsilon>0$ because $2-\varepsilon / r \geqslant(2-\varepsilon)-\varepsilon / r$

$\Lambda_{r 1}^{2-\varepsilon}\left(B^{3}, S^{2}\right) \subset H^{1}\left(B^{3}, S^{2}\right)$ for $r>1$ and $\varepsilon>0$ such that $(2-\varepsilon)-r / 3 \geqslant 1 / 2$. (All the inclusions are continuous).

If $r=3 /(2+\delta)$ with $1>\delta>0$ and $\varepsilon+\delta<1 / 2$ in order to have $(2-\varepsilon)-r / 3 \geqslant-1 / 2$, this condition implies also that $r \geqslant 2 /(2-\varepsilon)$. Therefore suppose that $\left\{u_{n}\right\}$ is a sequence of $C^{\infty}\left(B^{3}, S^{2}\right)$ converging to $x /|x|$ in $\Lambda_{r i}^{2-8}\left(B^{3}, S^{2}\right)$. By continuous injection from this last space on $H^{1}\left(B^{3}, S^{2}\right)$ the sequence $\left\{u_{n}\right\}$ should be convergent to $x /|x|$ also in this last space what is imposible.

As in the proof of proposition 1.5, for proving theorem 2.6 we have needed $M$ greater than $\max (s, 1)$. $p$. So we can not give a general density result as (2.6) when $0<s<1$. (In that case with exactly the same proof as in (2.6), we can prove the density only when $1 \leqslant p<M$ ). Nevertheless we can prove the following:

Theorem 2.11. If $p$ and $q$ are no less than one, for any integers $N \geqslant 1$ and $M \geqslant 1$ and any $s$ in $(0,1 / p)$ such that $1+N / p>s+N / q$ the set $C\left(\Omega, S^{s}\right)$ is dense in $\Lambda_{p q}^{s}\left(\Omega, S^{M}\right)$.

We shall prove this result in two steps. First showing that the set of step functions on $\Omega$ taking their values on $S^{M}$ is dense in $\Lambda_{p q}^{s}\left(\Omega, S^{M}\right)$. We conclude using stereographic projection as above.

The first step will be donne with three simple lemmas.

Lemma 2.12. If $s \in(0,1 / p)$, for any $N \geqslant 1$ and $M \geqslant 1$, the characteristic function of a cube $Q$ of $\mathbb{R}^{N}$ belongs to $\Lambda_{p q}^{s}\left(\mathbb{R}^{N}\right)$.

Proof. Using the characterisation of $\Lambda_{p q}^{s}$ for $0<s<1$ given in [9] it is an elementary calculus to see that if $Q=I_{1} \times I_{2} \times \ldots \times I_{N}$ where the $I_{j}$ are intervals of $\mathbb{R}$ whith Lebesgue measure $L_{j}$

$$
\left\|\chi_{Q}\right\|_{s, p, q} \leqslant C(N, s, p, q) \sum_{i=1}^{N} L_{1}^{1 / p}, L_{2}^{1 / p} \ldots L_{i}^{-s+(1 / p)} \ldots L_{N}^{1 / p}
$$

where $\chi_{Q}$ is the characteristic function of $Q$.

Lemma 2.13. Under the hypothesis of theorem 2.11 the set of finite and linear combinations of characteristic functions of cubes contained in $\Omega$, with vectorial coefficients belonging to $\mathbb{R}^{M+1}$ is dense in $\Lambda_{p q}^{s}\left(\Omega, S^{M}\right)$. 
Proof. Given any function $u$ of $\Lambda_{p d}^{s}\left(\Omega, S^{M}\right)$ it is well known that for any $\varepsilon>0$ fixed there is a function $v$ of $C^{\infty}\left(\Omega, B^{M}(0,1)\right)$ such that: $\|u-v\|_{\text {s,p; } q} \leqslant \varepsilon$.

Now it is easy, using diadic cubes in $\mathbb{R}^{N}$, to construct a sequence $\left\{v_{j}\right\}$ of step functions of the form:

$$
v_{j}(x)=\sum_{k \in K_{j}} \cdot v\left(x_{j, k}\right) \chi_{j, k}
$$

(where $\chi_{j, k}$ is the characteristic function of $Q(j, k)$ the $k-t$ th cube of the $j-t h$ generation, $x_{j, k}$ belongs to $Q(j, k)$ and $\# K_{j}$ is of order $2^{N_{j}}, \Omega \subset Q(0,0)$ ) such that:

$$
\left\|v_{j}-v_{j+1}\right\|_{s, p ; q}^{q} \leqslant C(\Omega, v, s, p, q) 2^{-j \cdot\left(-q s+\frac{q^{N}}{p}+q-N\right)}
$$

We deduce from this inequality and the hypothesis that $\left\{v_{j}\right\}$ is a Cauchy sequence in $\Lambda_{p q}^{s}$ (int $Q(0,0)$ ). Then defining $u_{j}=v_{j}\left[\Omega,\left\{u_{j}\right\}\right.$ is a Cauchy sequence in $\Lambda_{p q}^{s}(\Omega)$. As for almost every $x$ of $\Omega: u_{j}(x) \rightarrow u(x),\left\{u_{j}\right\}$ converges to $v$ in $\Lambda_{p q}^{s}(\Omega)$ and this ends the proof.

Lemma 2.14. Under the same hypothesis that in theorem 2.12 the set of step functions defined on $\Omega$ and taking their values on $S^{M}$ is dense in $\Lambda_{p q}^{s}\left(\Omega, S^{M}\right)$.

Proof. Let $u$ be any function of $\Lambda_{p q}^{s}\left(\Omega, S^{M}\right)$. By the above lemma we know that there is a sequence of step functions $\left\{v_{n}\right\}$ from $\Omega$ to $B(0,1)$ converging to $u$ in $\Lambda_{p q}^{s}\left(\Omega, S^{M}\right)$. We can write

$$
v_{n}(x)=\sum_{k \in K_{n}} \xi_{n, k} \chi_{n, k}(x)
$$

where $\xi_{n, k} \in B(0,1)$. Let $\Phi$ be a $C^{1}$ non negative function from $B(0,1)$ into itself such that $\Phi(x)=|x|$ for any $x$ of $B(0,1)$ such that $|x| \geqslant 1 / 4$ and $|\Phi(x)| \leqslant 1 / 4$ if $|x| \leqslant 1 / 4$. Define now:

$$
w_{n}=\sum_{k^{\prime} \in K^{\prime}} \xi_{n, k^{\prime}} \chi_{n, k^{\prime}}+\sum_{k^{\prime \prime} \in K^{\prime \prime}} \zeta_{0} \chi_{n, k^{\prime \prime}} \quad \text { where } \zeta_{0} \in S^{M} \text { is fixed }
$$

With $K^{\prime}=\left\{k^{\prime} ; 1-\left|\xi_{n, k^{\prime}}\right|<1 / 2\right\}$ and $K^{\prime \prime}=\left\{k^{\prime \prime} ;|1-| \xi_{n, k^{\prime \prime}}||<1 / 2\right\}$

Let us prove that $\left\{w_{n}\right\}$ still tends to $u$ in $\Lambda_{p q}^{s}(\Omega, B(0,1))$ :

$$
\begin{gathered}
\left\|u-w_{n}\right\|_{s, p ; q}^{q} \leqslant C\left\|u-v_{n}\right\|\left\|_{s, p ; q}^{q}+\right\| v_{n}-w_{n} \|_{s, p, q}^{q} \\
\leqslant C\left\|u-v_{n}\right\|_{s, p ; q}^{q}+C|| \sum_{k^{\prime \prime} \in K^{\prime \prime}}\left(\xi_{n, k^{\prime \prime}}-\zeta 0\right) \chi_{n, k^{\prime \prime}} \|_{s, p ; q}^{q} . \\
\leqslant C\left\|u-v_{n}\right\|_{s, p ; q}^{q}+C \sum_{k^{\prime \prime} \in K^{\prime \prime}}\left\|\chi_{n, k^{\prime \prime}}\right\|_{s, p ; q}^{q}
\end{gathered}
$$


Using now the results of section 1 it is clear that $\Phi_{0} w_{n}$ tends to one in $\Lambda_{p q}^{s}(\Omega, B(0,1))$. So we have:

$$
\begin{aligned}
\left\|1-\Phi_{0} w_{n}\right\|_{s, p ; q}^{q} & \geqslant C \sum_{k^{\prime \prime} \in K^{\prime \prime}}\left|1-\Phi\left(\xi_{n, k^{\prime \prime}}\right)\right|^{q}\left\|\chi_{n, k^{\prime \prime}}\right\|_{s, p, q}^{q} \geqslant . \\
& \geqslant C 2^{-q} \sum_{k^{\prime \prime} \in K^{\prime \prime}}\left\|\chi_{n, k^{\prime \prime}}\right\| \|_{, p, q}^{q}
\end{aligned}
$$

Therefore:

$$
\left\|u-w_{n}\right\|_{s, p ; q}^{q} \leqslant C\left\|u-v_{n}\right\|_{s, p . q}^{q}+C 2^{q}\left\|1-\Phi_{0} w_{n}\right\|_{s, p, q}^{q}
$$

and $\left\{w_{n}\right\}$ tends to $u$ as $n$ goes to $+\infty$. Observe that all the functions $w_{n}$ take their values on $B(0,1) \backslash B(0,1 / 2)$ because by construction of $\Phi, \Phi(x) \geqslant 1 / 2$ implies $|x| \geqslant 1 / 2$. Letting now $u_{n}=\operatorname{Proj} s^{2} w_{n}$ we have the result.

\section{NON DENSITY RESULTS}

As we said in the introduction, F. Bethuel and X. Zheng have proved that if $\mathfrak{N}$ is a compact Riemann manifold of dimension $M$ imbedded in $\mathbb{R}^{\gamma}, p<N$ and $\pi_{[p]}(\mathfrak{N}) \neq\{0\}$ (where $\pi_{[p]}(\mathfrak{N})$ is the [p]-homotopy group of $\mathfrak{M}$ ) then $C\left(B^{N}(0,1), \mathfrak{N}\right)$ is not dense in $W^{1, p}\left(B^{N}(0,1), \mathfrak{r}\right)$ (see [4] and [5]). We extend this result to the following cases:

Theorem 3.1. i) Let $1 \leqslant p<\infty, 1 \leqslant q<\infty, m \in \mathbb{N}$ such that $m p<N$ and $\pi_{[m p]}(\mathfrak{N}) \neq\{0\}$. Then $\left.C\left(B^{N}(0,1)\right), \mathfrak{R}\right) \cap W^{m, p}\left(B^{N}(0,1), \mathfrak{N}\right)$ is not dense in $W^{m, p}\left(B^{N}(0,1), \mathfrak{R}\right)$.

ii) If $1 \leqslant p<\infty, 1 \leqslant q<\infty, s>0$ are such that $s p<N$ and there are $s^{\prime}>0$, $p^{\prime} \geqslant 1, q^{\prime} \in\left[1, p^{\prime}\right]$ for which $[s p]=\left[s^{\prime} p^{\prime}\right], \Lambda_{p q}^{s}\left(B^{N}(0,1), \mathfrak{N}\right) \subset \Lambda_{p^{\prime} q^{\prime}}^{s^{\prime}}\left(B^{N}(0,1), \mathfrak{N}\right)$ and $\pi_{[s p]}(\mathfrak{N}) \neq\{0\}$ then $C\left(B^{N}(0,1), \mathfrak{R}\right) \cap \Lambda_{p q}^{s}\left(B^{N}(0,1)\right.$, $\left.\mathfrak{N}\right)$ is not dense in $\Lambda_{p q}^{s}\left(B^{N}(0,1), \mathfrak{M}\right)$.

In order to prove this theorem we give before two propositions relating convergence in Sobolev or Besov spaces and homotopy properties.

Proposition 3.2. Let $\mathfrak{M}$ be a compact Riemann manifold of dimension $N$, $\mathfrak{M}$ a compact Riemann submanifold of $\mathbb{R}^{M+1}$ and $g: \mathfrak{M} \rightarrow \mathbb{R}^{M+1}$ a $W^{m, p}$ map such that $g(x)$ belongs to $\mathfrak{N}$ for almost every $x$ of $\mathfrak{M}$ and $m p<N$. There is an $\varepsilon>0$ such that, if $f_{1}$ and $f_{2}$ from $\mathfrak{M}$ to $\mathfrak{N}$ are functions of $W^{m, p}$ and $\left\|f_{i}-g\right\|_{m, p}<\varepsilon(i=1,2)$ then $f_{1}$ and $f_{2}$ are [mp]-homotopic. 
Proof. Since $\mathfrak{R}$ is bounded in $\mathbb{R}^{M+1}$, the maps $g, f_{1}$ and $f_{2}$ belong to $L^{\infty}$. Then by the Gagliardo and Nirenberg's inequalities:

$$
\left\|f_{i}-g\right\|_{1, m p} \leqslant C\left\|f_{i}-g\right\|_{m, p}\left\|f_{i}-g\right\|_{\infty} \leqslant C\left\|f_{i}-g\right\|_{m, p} \text {, for } i=1,2,
$$

By Theorem 2 of $[15]$, there is an $\varepsilon>0$ such that $\left\|f_{i}-g\right\|_{1, m p} \leqslant \varepsilon(i=1,2)$ implies that $f_{1}$ and $f_{2}$ are $[\mathrm{mp}]$-homotopic.

Proposition 3.3. Let $\mathfrak{N}$ be a compact Riemann submanifold of $\mathbb{R}^{M+1}$. Let $g$ be a $\Lambda_{p q}^{s}$ map from $B^{N}(0,1)$ into $\mathfrak{N}$ whith $s p<N$ and $q \leqslant p$. There is an $\varepsilon>0$ such that if $f_{1}$ and $f_{2}$, from $B^{N}(0,1)$ to $\mathfrak{N}$ are in $\Lambda_{p q}^{s}$ and $\left\|f_{i}-g\right\|_{s, p ; q}<\varepsilon(i=1,2)$ then $f_{1}$ and $f_{2}$ are [sp]-homotopic.

Proof. Let $\left\{f_{i}\right\}$ be a sequence of $\Lambda_{p q}^{s}$ maps from $B^{N}(0,1)$ into $\mathfrak{N}$ such that $\left\|f_{i}-g\right\|_{s, p: q} \leqslant 2^{-i}$. Since $q \leqslant p, \Lambda_{p q}^{s} \subset \Lambda_{p p}^{s}$ with continuity. On the other hand since $\mathfrak{R}$ is bounded the functions $f, f_{1}$ and $f_{2}$ are bounded and then for $r=\min (s, s /([s]+1))$ :

$$
\left\|f_{i}-g\right\|_{r, s p l ; ; s p ; r} \leqslant C|| f_{i}-\left.g\right|_{, p, p ; p} \leqslant C\left\|f_{i}-g\right\|_{s, p ; q} \leqslant C 2^{-i}
$$

Using elementary properties of Lebesgue integral on $\mathbb{R}^{N}$ and the same arguments as in [15] we obtain that there are $r_{0} \in(0,1 / 2), t_{1} \in(-1,1)$ and for $i=2, \ldots, m$ (where $m=N-[s p]-1$ ),

$$
t_{i} \in\left(-\sqrt{1-t_{1}^{2}} \ldots \sqrt{1-t_{i-1}^{2}}, \sqrt{1+t_{1}^{2}} \ldots \sqrt{1+t_{i-1}^{2}}\right)
$$

such that, if

$$
\begin{gathered}
X=\left\{x^{\prime} \in \mathbb{R}^{[s p]+1} ; \sum_{i=1}^{[s p]+1} x_{i}^{2}+\sum_{i=1}^{m} t_{i}^{2}=r_{0}\right\} \quad \mathbf{t}=\left(t_{1}, \ldots, t_{m}\right) \\
\exists C>0 ; \forall k \in \mathbb{N} \int_{X}\left(\int_{B^{N}(0,1)}\left|f_{k}\left(x^{\prime}, \mathbf{t}\right)-f_{k}(y)\right|^{s p / r} \frac{d y}{\left.\mid\left(x^{\prime}, \mathbf{t}\right)-y\right)\left.\right|^{N+s p}}\right) d \sigma\left(x^{\prime}\right) \leqslant C \\
\lim _{k \rightarrow \infty} \int_{X}\left|f_{k+1}\left(x^{\prime}, \mathbf{t}\right)-f_{k}\left(x^{\prime}, \mathbf{t}\right)\right|^{s p p r} d \sigma\left(x^{\prime}\right)=0
\end{gathered}
$$

We conclude with the same tools that in [15] using that the [sp]-skeleton of $B^{N}(0,1)$ is $S^{[s p]}$.

Proof of 3.1. i) Let $P_{[m p]+1}$ be the projection $\mathbb{R}^{N}$ to $\mathbb{R}^{[m p]+1}$ and $\pi$ radial projection from $\mathbb{R}^{[m p]+1}$ to $S^{[m p]}$. Define the function $g=\pi_{0} P_{[m p]+1}$. We have that $g \in W^{m, p}\left(B^{N}(0,1), S^{[m p]}\right)$ and $g_{\mid s^{m p]}}=I d$.

On the other hand, since $\Pi_{[m p]}(\mathfrak{N}) \neq\{0\}$ there is a $C^{[m p]+1}$ map, $\Phi$ from $S^{[m p]}$ to $\mathfrak{N}$ which can not be extended continuously to $B^{[m p]+1}$. Let us define 
$f=\Phi$ og. By the results of section $1, f \in W^{m, p}\left(\left(B^{N}(0,1), \mathfrak{N}\right)\right.$. Now suppose there is a sequence $\left\{f_{n}\right\}$ of functions of $C\left(\left(B^{N}(0,1), \Re\right)\right.$ converging to $f$ in $W^{m, p}\left(\left(B^{N}(0,1), \Re\right)\right.$. By proposition (3.2) $f_{n}$ and $f$ are [mp]-homotopic for $n$ large enough. Since $f_{n}$ is smooth on $B^{N}(0,1)$, by the homotopy extension theorem we may extend $f$ to $B^{N}(0,1)$ continuously but that is impossible by construction.

ii) Let us define the function $f$ in the same way as above, with $[s p]$ instead of $[m p]$. Suppose again that there is a sequence of continuous functions $\left\{f_{n}\right\}$ converging to $f$ in $\Lambda_{p q}^{s}\left(B^{N}(0,1), \mathfrak{M}\right)$. By the hypothesis $\left\{f_{n}\right\}$-converges to $f$ in $\Lambda_{p^{\prime}}^{s^{\prime}},\left(B^{N}(0,1) ; \mathfrak{R}\right)$. The proof follows now as above.

Remark 3.4. Using the inclusions recalled in the first section it is very simple to see that the conditions in ii) of Theorem (3.1) are satisfied is $s \cdot p$ is not an integer and $\Pi_{[s p]}(\mathfrak{i}) \neq\{0\}$. If $s p$ is an integer we must to have $q \leqslant p$.

\section{REFERENCES}

[1] ADAms, R.: Sobolev Spaces, Academic Press (1975).

[2] BREZIS, H.: Large harmonic maps in two dimensions. Non linear variational problems. Research notes in Mathematic. Pitman, $n^{0} 127$.

[3] BREZIs, H.: Analyse fonctionelle. Théorie et applications. Masson (1983).

[4] BETHUEL, $F$. and ZHENG, $X$.: Sur la densité des fonctions régulières entre deux variétés dans des espaces de Sobolev, C. R. Acad. Sci., Paris (1986).

[5] BETHueL, F. and ZHENG, X.: Density of smooth functions between two manifolds in Sobolev spaces, J. Funct. Anal. (to appear).

[6] Dorronsoro, J. R.: Mean oscillation and Besov spaces, Canad. Math. Bull., vol. 28(4), 1985.

[7] Dorronsoro, J. R.: A characterisation of potential spaces, Proc. Amer. Marh. Soc., vol. 95 (1), 1985.

[8] Friedman, A.: Partial differential equations, Holt-Rinehart-Winston (1969).

[9] LIONS, J. L., and PEETRE, J.: Sur une classe d'espaces d'interpolation. Inst. Hautes Etudes, 19, Paris (1964).

[10] SCHOEN, R. and UhLENBeCK, K.: Boundary regularity, and the Dirichlet problem for harmonic maps, J. Diff. Geom., 18 (1983), 253-268.

[11] SCHOEN, R. and UHLENBECK, K.: A' regularity theory for harmonic maps, $J$. Diff. Geom., 17 (1982), 307-335.

[12] SCHOEN, R. and UHLENBECK, K.: Approximation theorems for Sobolev mappings. Preprint.

[13] SImon, J., Sur des équation aux dérivées partielles non linéaires, Thèse de Doctorat d'Etat. Université P. and M. Curie, 1977.

[14] TRIEBEL, H., Theory of Function Spaces, Birkhauser Verlag (1983).

[15] WHITE, B.: Infima of energy functionals in homotopy classes of mappings, $J$, Diff. Geometry, 23 (1986), 127-142. 